\section{Case Reports in Dermatology}

\title{
M2-Polarized Macrophages Compose Lupus Vulgaris Arising from a Bacillus Calmette-Guérin Vaccination Site
}

\author{
Yota Sato $^{a} \quad$ Taku Fujimura $^{a} \quad$ Sadanori Furudate $^{a} \quad$ Aya Kakizaki $^{a}$ \\ Osamu Iizawa $^{\mathrm{b}}$ Setsuya Aiba ${ }^{\mathrm{a}}$ \\ aDepartment of Dermatology, Tohoku University Graduate School of Medicine, \\ Sendai, Japan; ${ }^{b}$ Department of Dermatology, Sendai Medical Center, Sendai, Japan
}

\section{Keywords}

M2-polarized macrophages · Lupus vulgaris · Bacillus Calmette-Guérin · Immunosuppression

\begin{abstract}
Since bacillus Calmette-Guérin (BCG) is an attenuated strain of Mycobacterium bovis, lupus vulgaris (LV) is reported as one of the rare complications after BCG vaccination, correlating with immunosuppression in the lesional skin. In this report, we describe a case of LV arising from the BCG vaccination site 22 years after vaccination. Interestingly, in the present case, granuloma cells were composed of M2-polarized macrophages. Our case might explain the contribution of M2-polarized macrophages to the biology of LV arising from a BCG vaccination site.

\section{Introduction}

Bacillus Calmette-Guérin (BCG) is an attenuated strain of Mycobacterium bovis [1, 2]. Since BCG is usually considered to be avirulent, it is used for vaccination against tuberculosis in Japan for infants. On the other hand, a previous report suggested that delayed granuloma formation could develop at the BCG vaccination site, though the granuloma-composing cells 
were still unknown [1-5]. In this report, we describe a case of lupus vulgaris (LV) arising from a BCG vaccination site 22 years after vaccination which was composed of M2-polarized macrophages.

\section{Case Report}

A 64-year-old Japanese man visited our outpatient clinic with a 30-year history of pruritic erythema on the left arm. His condition had been treated in a private clinic with topical steroids, and he consulted our hospital for metastatic skin cancer. He had been given BCG vaccination into the left arm when 12 years old. On his initial visit, physical examination revealed erosive, asymptomatic nodules developing from a well-defined erythematous plaque on the left arm (fig. 1a). A biopsy specimen showed non-caseating granulomas with epithelioid cells (fig. 1b, c). Cultures on 2\% Ogawa medium detected the Mycobacterium tuberculosis complex, leading to identification of $M$. bovis BCG strain Tokyo 172. From the above finding, we diagnosed this patient as LV arising from the BCG vaccination site. We administered oral isoniazid $300 \mathrm{mg}$ per day for 3 months. Consequently the nodule disappeared, leaving a scar. One year after stopping isoniazid, there was no sign of relapsing nodules.

Since M. bovis BCG has been reported to induce M1 macrophages [6] and, in contrast, M2-polarized macrophages have been reported to contribute to tissue remodeling [7], we employed immunohistochemical staining for CD68 (fig. 2a), iNOS (fig. 2b), CD163 (fig. 2c), CD206 (fig. 2d) and periostin (fig. 2e, f) to investigate the immunological background of the granuloma tissue. The granuloma-composing macrophages were mainly composed of CD68+ cells, CD163+ macrophages and CD206+ cells. Few iNOS-expressing cells were detected in the present case (fig. 2b). Periostin was prominent in the stroma of granulomas adjacent to CD163+ macrophages (fig. 2f).

\section{Discussion}

The frequency of LV as one of the rare complications after BCG vaccination [2-4] is estimated to be only 5 per 1 million vaccinations $[2-4,8]$. $\mathrm{LV}$ is a chronic form of tuberculosis occurring in individuals with moderate to high immunity against tubercle bacilli. Since LV induced by BCG vaccination is correlated with immunosuppression such as cellular immune defects and impaired IL-12- and interferon- $\gamma$-mediated immunity [9], BCG-induced LV might complicate skin cancer, including squamous cell carcinoma and basal cell carcinoma $[1,10]$. Notably, these skin cancers contain tumor-associated macrophages in the lesional skin to maintain an immunosuppressive tumor microenvironment together with regulatory $\mathrm{T}$ cells [11-13]. In addition, von Bubnoff et al. [11] reported that infectious cutaneous granulomas, including conventional LV, possess granulomas composed of CD68+ macrophages that express indoleamine 2,3-dioxygenase, which degrades the tryptophan in the surrounding microenvironment to suppress $\mathrm{T}$ cell function. From the above reports, we hypothesized that BCG-induced LV might possess M2-polarized macrophages that contributed to the development of LV.

To prove our hypothesis, we employed immunohistochemical staining for CD68 (a common myeloid marker), iNOS (M1 marker), CD163 and CD206 (M2 markers), as well as periostin, which was previously reported to stimulate CD163+ macrophages to produce 
various functional factors such as chemokines and matrix metalloproteinases [14]. As expected, the granuloma cells were mainly composed of CD68+ myeloid cells, CD163+ macrophages and CD206+ cells. In addition, periostin was prominent in the stroma of granulomas, suggesting that periostin might stimulate CD163+ macrophages to establish the granuloma by producing various chemokines [14]. Since this report presents only a single case, further analysis of the mechanisms underlying this phenomenon may provide fundamental insights into the biology of LV arising from a BCG vaccination site. Such issues will need to be clarified in future investigations.

\section{Statement of Ethics}

The patient gave written informed consent.

\section{Disclosure Statement}

The authors declare no conflict of interest.

\section{References}

1 Thakur BK, Verma S: BCG-induced lupus vulgaris complicated by squamous cell carcinoma in a 7-yearold child. Int J Dermatol 2011;50:542-545.

2 Walker SL, Lozewicz S, Sood R, Mann TA, Campalani E, Hubbard VG: Lupus vulgaris due to Mycobacterium bovis bacillus Calmette-Guérin (BCG) at the site of previous BCG vaccination. Clin Exp Dermatol 2009;34:e213-e215.

-3 Lo Schiavo A, Brasiello M, Sangiuliano S, Ruocco E: Tuberculosis and cancer on a BCG vaccination site: an instance of immunocompromised district. Int J Dermatol 2014;53:e28-e29.

4 Farsinejad K, Daneshpazhooh M, Sairafi H, Barzegar M, Mortazavizadeh M: Lupus vulgaris at the site of BCG vaccination: report of three cases. Clin Exp Dermatol 2009;34:e167-e169. Attia E: BCG vaccine-induced lupus vulgaris. Eur J Dermatol 2007;17:547-548.

Kumar P, Tyagi R, Das G, Bhaskar S: Mycobacterium indicus pranii and Mycobacterium bovis BCG lead to differential macrophage activation in Toll-like receptor-dependent manner. Immunology 2014;143: 258-268.

7 Gordon S, Martinez FO: Alternative activation of macrophages: mechanism and functions. Immunity 2010;32:593-604.

8 Handjani F, Delir S, Sodaifi M, Kumar PV: Lupus vulgaris following bacille Calmette-Guérin vaccination. Br J Dermatol 2001;144:444-445.

-9 Döffinger R, Dupuis S, Picard C, Fieschi C, Feinberg J, Barcenas-Morales G, Casanova JL: Inherited disorders of IL-12- and IFNgamma-mediated immunity: a molecular genetics update. Mol Immunol 2002;38:903-909.

10 Polat M, Parlak AH, Hasdemir O, Boran C: Basal cell carcinoma in a bacillus Calmette-Guérin scar. Int J Dermatol 2009;48:542-543.

-11 von Bubnoff D, Scheler M, Wilms H, Wenzel J, von Bubnoff N, Häcker G, Schultze J, Popov A, Racz P, Bieber T, Wickenhauser C: Indoleamine 2,3-dioxygenase-expressing myeloid dendritic cells and macrophages in infectious and noninfectious cutaneous granulomas. J Am Acad Dermatol 2011;65: 819-832.

12 Fujimura T, Kakizaki A, Furudate S, Kambayashi Y, Aiba S: Tumor-associated macrophages in skin: how to treat their heterogeneity and plasticity. J Dermatol Sci DOI: 10.1016/j.jdermsci.2016.05.015.

13 Kambayashi Y, Fujimura T, Aiba S: Comparison of immunosuppressive cells and immunomodulatory cells in keratoacanthoma and invasive squamous cell carcinoma. Acta Derm Venereol 2013;93:663668.

14 Furudate S, Fujimura T, Kakizaki A, Kambayashi Y, Asano M, Watabe A, Aiba S: The possible interaction between periostin expressed by cancer stroma and tumor-associated macrophages in developing mycosis fungoides. Exp Dermatol 2016;25:107-112. 


\section{Case Reports in \\ Dermatology}

Case Rep Dermatol 2016;8:202-206

(C) 2016 The Author(s). Published by S. Karger AG, Basel www.karger.com/cde

Sato et al.: M2-Polarized Macrophages Compose Lupus Vulgaris Arising from a Bacillus Calmette-Guérin Vaccination Site

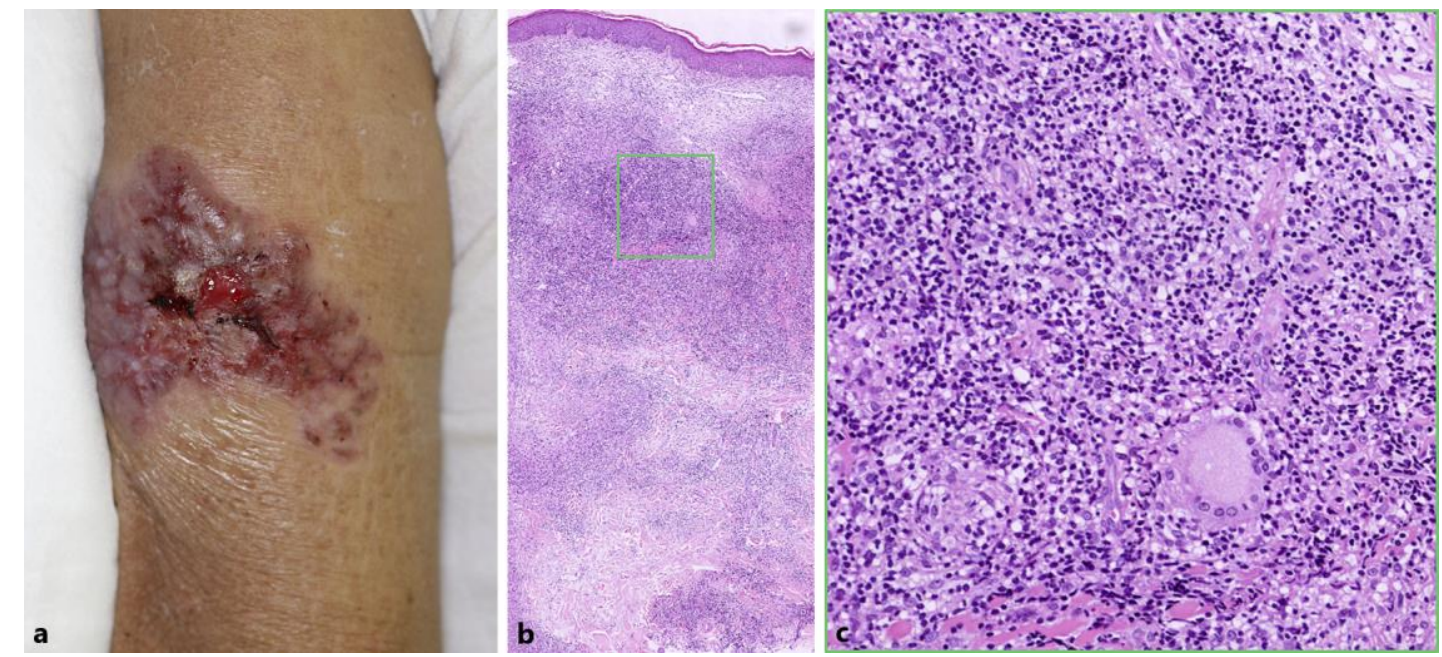

Fig. 1. a Erosive, asymptomatic nodules developing from a well-defined erythematous plaque on the left arm. b, c Non-caseating granulomas with epithelioid cells were detected throughout the dermis. Original magnification: $\times 100($ b),$\times 400$ (c). 


\section{Case Reports in \\ Dermatology}

Case Rep Dermatol 2016;8:202-206

(C) 2016 The Author(s). Published by S. Karger AG, Basel
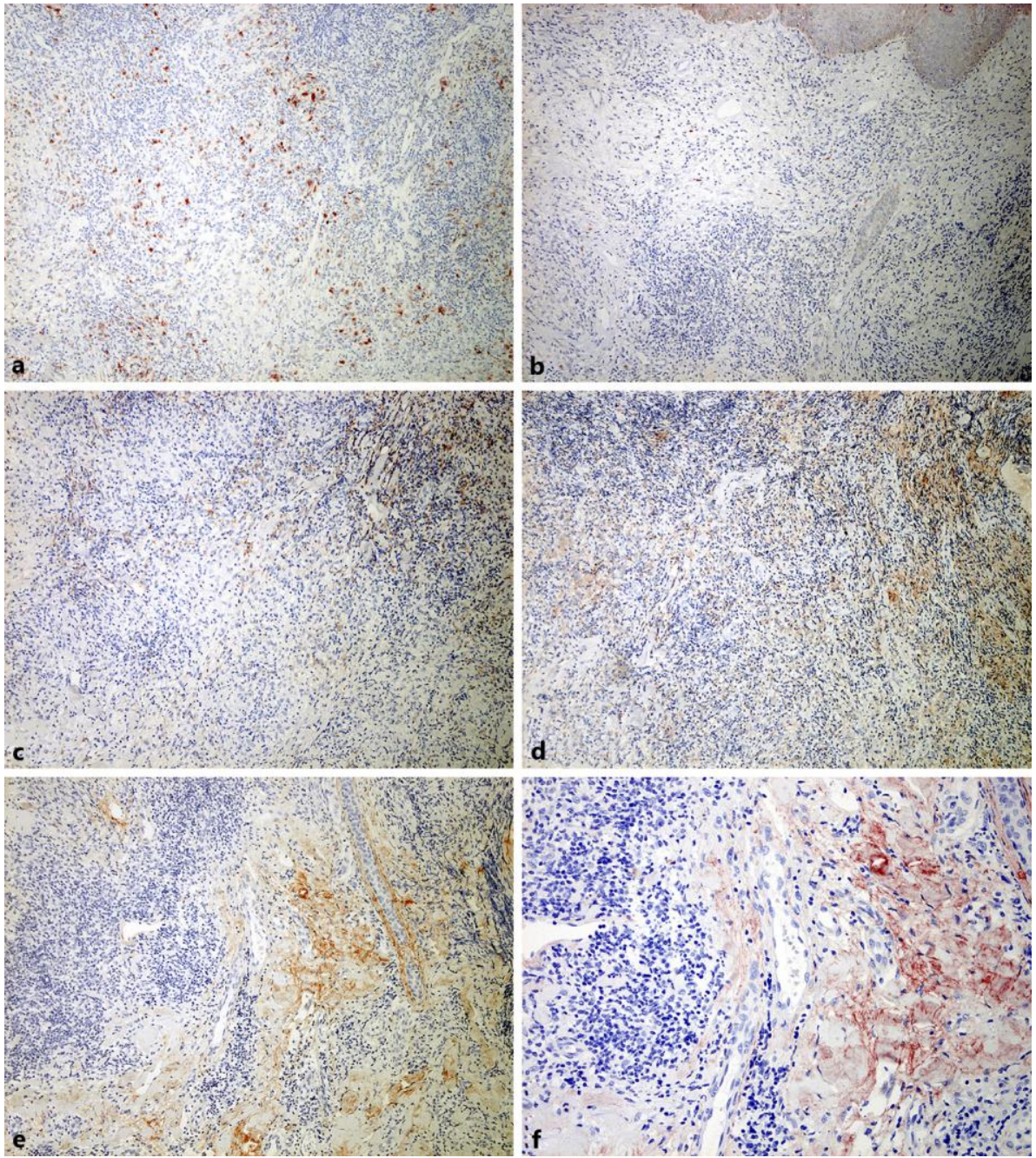

Fig. 2. Paraffin-embedded tissue samples were deparaffinized and stained with anti-CD68 Ab (a), anti-iNOS $\mathrm{Ab}(\mathbf{b})$, anti-CD163 Ab (c), anti-CD206 Ab (d) and anti-periostin Ab (e, f). The sections were developed with liquid permanent red. Original magnification: $\times 100$ (a-e), $\times 200$ (f). 